\title{
Gravitational lensing along multiple light paths as a probe of physics beyond Einstein gravity
}

Hideki Asada*

Faculty of Science and Technology, Hirosaki University, Hirosaki 036-8561, Japan

E-mail: asada@phys.hirosaki-u.ac.jp

The light propagation is reexamined, allowing for various models of modified gravity. We clarify the dependence of the time delay (and induced frequency shift) on modified gravity models and investigate how to distinguish those models, when light propagates in static spherically symmetric spacetimes parameterized to express modified gravity. Implications to gravitational lensing are mentioned.

The Manchester Microlensing Conference: The 12th International Conference and ANGLES Microlensing Workshop

January 21-25 2008

Manchester, $U K$

${ }^{*}$ Speaker. 


\section{Introduction}

A certain modification, in whatever form, in the standard cosmological model, is strongly suggested by recent observations such as the magnitude-redshift relation of type Ia supernovae (SNIa) and the cosmic microwave background (CMB) anisotropy by WMAP. We are forced to add a new component into the energy-momentum tensor in the Einstein equation or modify the theory of general relativity itself. Indeed, plenty of models have been proposed, such as scalar tensor theories, string theories, higher dimensional scenarios and quantum gravity. Therefore, it is of great importance to observationally test these models.

The theory of general relativity has passed "classical" tests, such as the deflection of light, the perihelion shift of Mercury and the Shapiro time delay, and also a systematic test using the remarkable binary pulsar "PSR $1913+16$ ". In the twentieth century, these tests proved that the Einstein's theory is correct with a similar accuracy of $0.1 \%$.

The gravitational time delay effect along a light path has successfully tested the Einstein's theory, where the gravitational time delay is supplementary to the gravitational lensing via Fermat's principle. A significant improvement was reported in 2003 from Doppler tracking of the Cassini spacecraft on its way to the Saturn, with $\gamma-1=(2.1 \pm 2.3) \times 10^{-5}$ [2]. Here, $\gamma$ is one of parameters in the parameterized post-Newtonian (PPN) formulation of gravity. The sensitivity in the Cassini experiment approaches the level at which, theoretically, deviations $10^{-6}-10^{-7}$ are expected in some cosmological models [3] 4 . Therefore, it is important to investigate the Shapiro time delay with such a high accuracy. We shall discuss the dependence of the time delay (and induced frequency shift) on modified gravity models and investigate how to distinguish those models by using the Shapiro time delay ([1]; many references are therein). Introducing a new energy or length scale (e.g. extra dimension scale) may make changes in functional forms of the gravitational field. Thus it is worthwhile to investigate how to probe such a modified functional form, by using the light propagation. Throughout this paper, we take the units of $G=c=1$.

\section{Shapiro Time Delay}

Assumption: the electromagnetic fields propagate in four-dimensional spacetimes (even if the whole spacetime is higher dimensional). Thus photon paths follow null geodesics (as the geometrical optics approximation of Maxwell equation).

We shall consider a static spherically symmetric spacetime, in which light propagates, expressed as

$$
d s^{2}=-A(r) d t^{2}+B(r) d r^{2}+r^{2} d \Omega^{2},
$$

where $r$ and $d \Omega^{2}$ denote the circumference radius and the metric of the unit 2-sphere, respectively. The functions $A(r)$ and $B(r)$ depend on gravity theories.

The time lapse along a photon path is obtained as

$$
t\left(r, r_{0}\right)=\int_{r_{0}}^{r} \frac{d r}{b} \sqrt{\frac{B(r)}{A(r)}} \frac{1}{\sqrt{\frac{A\left(r_{0}\right)}{r_{0}^{2}}-\frac{A(r)}{r^{2}}}}
$$


where $b$ and $r_{0}$ denote the impact parameter and the closest point, respectively. Their relation is $b^{2}=r_{0}^{2} / A\left(r_{0}\right)$.

For practical calculations, we keep only the leading term at a few AU in the corrections. Namely, $A(r)$ and $B(r)$ are approximated as

$$
\begin{aligned}
& A(r) \approx 1-\frac{2 M}{r}+A_{m} r^{m} \\
& B(r) \approx 1+\frac{2 M}{r}+B_{n} r^{n}
\end{aligned}
$$

where $M$ denotes the mass of the central body.

Examples of modified gravity theories: (1) $n=1 / 2, A_{n}=-2 B_{n}=-2 \sqrt{M / r_{c}^{2}}$ for DGP model with $r_{c}$ that is the extra scale within which gravity becomes five dimensional. (2) $n=3 / 2$, $A_{n}=(2 / 3) m_{g}^{2} \sqrt{2 M / 13}$ and $B_{n}=-m_{g}^{2} \sqrt{2 M / 13}$ with graviton mass $m_{g}$ for one of massive gravity models. (3) $n=2, A_{n}=-B_{n}=-\Lambda / 3$ for the Schwarzschild-de Sitter spacetime, that is, general relativity with the cosmological constant $\Lambda$ as a possible candidate for the dark energy.

Up to the linear order, the extra contribution to time delay due to modified gravity is

$$
\begin{aligned}
\delta t= & r_{0}^{n+1}\left(\int_{1}^{R_{E}}+\int_{1}^{R_{R}}\right) d R \\
& \times\left(-A_{n} \frac{R^{n+3}-2 R^{n+1}+R}{\left(R^{2}-1\right)^{3 / 2}}+B_{n} \frac{R^{n+1}}{\sqrt{R^{2}-1}}\right),
\end{aligned}
$$

where we define $R \equiv r / r_{0}$.

It is convenient to use the relative change in the frequency, which is caused by the gravitational time delay. This frequency shift is defined as $y=-d(\Delta T) / d t$. For a receiver at $r_{R}>r_{E}$, the extra frequency shift is

$$
\begin{aligned}
\delta y & \sim\left(A_{n}+B_{n}\right) r_{R}^{n} \frac{b}{r_{R}} \frac{d b}{d t} \\
& \sim 10^{-17}\left(\frac{10 \mathrm{AU}}{r_{\odot}}\right)^{n}\left(\frac{\left(A_{n}+B_{n}\right) r_{\odot}^{n}}{10^{-10}}\right)\left(\frac{r_{R}}{10 \mathrm{AU}}\right)^{n-1}\left(\frac{b}{r_{\odot}}\right)\left(\frac{d b / d t}{v_{E}}\right),
\end{aligned}
$$

where $10 \mathrm{AU} / r_{\odot} \sim 2 \times 10^{3}$. The larger the index of $n$, the longer the delay $\delta y$.

Figure 1 shows that an extra distortion due to $\delta y$ would appear especially in the tail parts of $y-t$ curves. One can distinguish modified gravity models, which are characterized by various values of $n, A_{n}, B_{n}$, from observations using receivers at very different distances from Sun, as shown by Fig. 1

We consider three light paths, for which the impact parameters of the photon paths are almost the same (several times of the solar radius) for convenience sake. The locations of the receivers are denoted as $r_{R 1}, r_{R 2}$ and $r_{R 3}$, where the subscripts from 1 to 3 denote each light path. We assume that $r_{E}$ is constant in time for simplicity. It is a straightforward task to take account of the eccentricity of the Earth orbit and a difference between the impact parameters. It can be shown that $A_{n}+B_{n}$ and $n$ are separately determined by using at least three very different light paths [1]. 


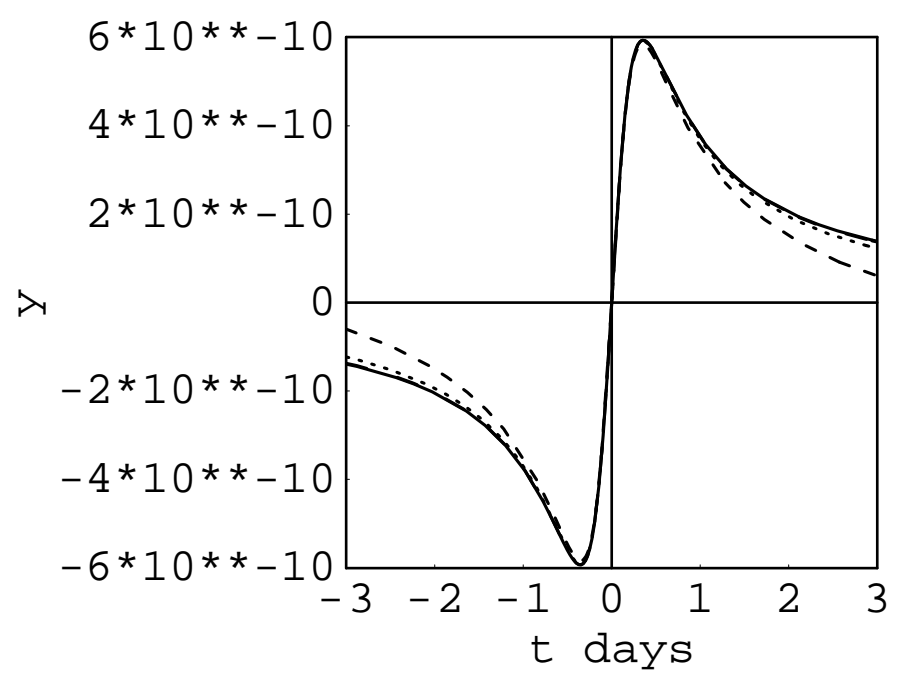

Figure 1: Dependence of the frequency shift on the distance $r_{R}$ and the index $n$. The long dashed, short dashed and dotted curves denote the frequency shift for $\left(n, r_{R}\right)=(3 / 2,10 \mathrm{AU}),\left(n, r_{R}\right)=(2,10 \mathrm{AU}),\left(n, r_{R}\right)=$ (2,1AU), respectively. The long dashed curve for $n=3 / 2$ and $r_{R}=10 \mathrm{AU}$ is overlapped with the solid curve denoting the general relativistic case. Here, we assume $\left(A_{n}+B_{n}\right) r_{\odot}^{n}=3 \times 10^{-11}$.

\section{Conclusion - Implications to gravitational lensing}

The present result suggests that the tail part of gravitational lensing (and time delay) seems sensitive to certain modifications to general relativity.

Statistical investigations of gravitational lensing with future micro-arcsec. astrometry missions such as SIM, GAIA and JASMINE will probably become a probe of gravity theories. For instance, the bending angle by the solar gravity is of the order of 1 mas even at a different direction $(\sim 90 \mathrm{deg}$.) from Sun. The number of source stars for those missions, $N$, is quite large, say $10^{6}$. Therefore, one can make a rather optimistic estimate of the possible constraint on modified gravity with the accuracy as

$$
\frac{1 \mu \text { as }}{1 \operatorname{mas}} \times \frac{1}{\sqrt{N}} \sim 10^{-7}
$$

More detailed investigations on gravitational lensing including microlensing as a probe of modified gravity would be important.

\section{References}

[1] H. Asada, accepted for publication in Phys. Lett. B. (2008) (arXiv:0710.0477 [gr-qc]).

[2] B. Bertotti et al. Nature, 425, 374 (2003).

[3] T. Damour and A. M. Polyakov, Nucl. Phys. B 423, 532 (1994);

[4] T. Damour, F. Piazza and G. Veneziano, PRD 66, 046007 (2002).

[5] A. I. Vainshtein, PLB 39, 393 (1972).

T. Damour, I. I. Kogan and A. Papazoglou, PRD 67, 064009 (2003). 